\title{
Numerical and Analytical Calculation of Flow During Steam Blowing
}

\author{
Michal Hozned1 ${ }^{1, *}$, and Karel Gregor ${ }^{2}$ \\ ${ }^{1}$ Doosan Škoda Power s.r.o., Experimental Research, Tylova 1/57, Pilsen, Czech Republic \\ ${ }^{2}$ Doosan Škoda Power s.r.o., Thermodynamics, Tylova 1/57, Pilsen, Czech Republic
}

\begin{abstract}
In the paper results are described of numerical simulations of the flow during the steam blowing between the boiler drum and the outlet to the atmosphere. Numerical flow simulations are compared to the analytical approach that best describes the flow during the blowing, i.e. the Fanno flow. The proposed methodology of analytical calculation can be, with a reasonable deviation from reality, used in control of velocity and flow in the pipe outlet cross-section.
\end{abstract}

\section{Introduction}

One of the activities before the first start of the steam turbine is the so-called steam blowing. The steam blowing cleans dirt in the pipeline from the steam pipe between the boiler drum up to the inlet into the control or quick-opening valve. In the pipeline there are solid particles generated by transport, grinding or welding of the pipeline. These particles could damage the valves or the turbine blades. Thus there is a bypass installed before the valves and temporary pipes allowing the steam to escape to atmosphere. At the end of the steam pipeline the target plate, made of aluminium or stainless steel is installed. During the blowing the boiler drum is connected with atmosphere and for certain steam velocity and for certain time the loose debris causes indents on the target plate. When the blow is terminated the target plate is taken out and the number of indents is counted. If the number and size of indents is below certain level, the blow can be considered successfully completed.

From the viewpoint of the steam flow around the plate it is necessary to preserve for a given period the CFR (Cleaning Force Ratio), defined by the equation:

$$
C F R=\frac{\dot{m}_{c}^{2} \cdot v_{c}}{\dot{m}_{n}^{2} \cdot v_{n}} \geq 1.2
$$

where:

$\dot{m}_{c}$ and $\dot{m}_{n}$ is the steam mass flow during the blow or in common operation, $v_{c}$ and $v_{n}$ is specific volume of steam during the blow or in common operation.

In some cases it is possible to apply the relation that uses only the steam velocities ratio at the plate location.

${ }^{*}$ Corresponding author: michal.hoznedl@doosan.com 
In principle, there are two methods of steam blowing: the continuous blow for which constant pressure must be maintained at all times in the steam boiler drum. However, this requires a higher amount of used water. The second possibility is to use the puffing method, when after opening the quick-opening valve the steam pressure in the drum drops to the saturation limit and water consumption is lower.

As it is only a temporary steam pipe, for financial reasons the number of measuring points is limited. Thus only pressure and temperature of the boiler drum are measured and atmospheric pressure is known. From these data it is necessary to define the steam mass flow to determine the CFR ratio.

The issue of steam blowing is rarely mentioned in research literature, and if so, it is rather about the concept of the pipeline system than about the flow itself [1], or the possibilities are discussed of lowering the CFR ratio $[2,3]$ using a more suitable design of the whole temporary pipeline.

\section{Calculation of velocity at the target plate location}

Many flow models are used for steam blowing in order to calculate the flow velocity at the target plate location. However, each method involves significant simplifying assumptions.

\subsection{Bernoulli equation}

In this case it is necessary to define friction and local losses along the blow line; however, they depend on an unknown velocity. The density is calculated as constant from the mean value of the pressure along the flow line and assuming a constant temperature. By combining Bernoulli equation and continuity equation the relation for flow can be obtained.

$$
\dot{m}=\sqrt{\frac{p_{1}^{2}-p_{2}^{2}}{r \cdot T \cdot\left(\sum \lambda \cdot \frac{L}{D}+\sum \zeta\right)}}
$$

Here: $p_{1}$ and $p_{2}$ are pressures in the steam drum, or at the target plate location,

$T$ is thermodynamic temperature in the steam drum,

$L$ is the length of the blow pipeline and $D$ its diameter,

$r$ is the specific gas constant,

$\lambda$ and $\zeta$ are friction or local losses in the pipeline respectively.

\subsection{Isothermal flow}

There is a type of flow used mainly on long pipelines. The temperature of gas (or another medium) is considered as constant throughout the volume and usually corresponds with the temperature of the ambient air, flow velocities are low. For this flow calculation friction losses are considered in horizontal pipeline of constant cross-section. These friction losses are constant along the pipeline.

$$
\dot{m}=\sqrt{\frac{p_{1}^{2}-p_{2}^{2}}{p_{1}} \cdot \frac{S^{2}}{v_{1} \cdot\left(\lambda \cdot \frac{L}{D}+2 \cdot \ln \frac{p_{1}}{p_{2}}\right)}}
$$


Here: $v_{l}$ is specific volume at the pipeline inlet and $S$ is the pipeline cross section area. The equitation is thus only suitable when the pressure drop caused by losses is important and linear along the piping length.

\subsection{Defining the amount of blow steam based on water loss in the drum}

This is an indirect determination of flow rate in case the steam drum is fitted with a level gauge and it is possible to read the water level in the drum provided the weight of the supplied feed water is known. It is possible to determine the flow rate through the blow pipeline:

$$
\dot{m}=\frac{F}{\Delta t}-\frac{\Delta m}{\Delta t}
$$

Here: $F$ is the weight of the supplied feed water during the time difference $\Delta t$ and $\Delta m$ is the change in the weight change of saturated steam and saturated water defined from the water level height. It is conceptually an exact method of defining the flow rate provided that level calibration as a function of the drum volume is available. However, this can be only guaranteed in exceptional cases.

\subsection{Fanno flow}

Another possibility to approach the steam flow calculation is the so called Fanno flow. It is the flow in the pipeline with a constant area, adiabatic walls and velocities that at the pipeline outlet reach the values of Mach number $M a=1$. Where pipe diameter $D$ and pipe length $L$ and also the pressure and temperature in the steam drum are known, by using this way of calculation it is possible to examine the distribution of pressure, temperature as well as sound and flow velocity. It is also possible to calculate density, specific volume, Reynolds number and thus also the friction coefficient $\lambda$ along the pipeline. In principle, it is also possible to define the flow rate anywhere in the pipeline even if this rate is approximately constant along the pipeline and the deviation is only due to inaccuracies in the calculation. First of all it is necessary to calculate Mach number $M a_{l}$ at the pipe inlet:

$$
L_{1}=\frac{D}{\lambda_{1} \cdot \kappa} \cdot\left[\frac{1-M a_{1}^{2}}{M a_{1}^{2}}+\frac{\kappa+1}{2} \cdot \ln \frac{(\kappa+1) \cdot M a_{1}^{2}}{2+(\kappa-1) \cdot M a_{1}^{2}}\right]
$$

Here: $\kappa$ is isentropic coefficient (for overheated steam $\kappa=1.33$ ) and $L_{l}$ is the total length of the pipeline.

Then it is necessary to determine at the pipeline inlet the speed of sound, flow velocity, density and dynamic viscosity using water steam tables.

In the next step equation (5) is used to define Mach number at any place along the pipeline. Knowing $M a_{1}$ at the pipeline inlet and $\mathrm{Ma}$ at any place it is possible to determine the temperature at any place along the pipeline:

$$
t=t_{1} \cdot \frac{(\kappa-1) \cdot M a_{1}^{2}+2}{(\kappa-1) \cdot M a^{2}+2}
$$

And also pressure at any place: 


$$
p=p_{1} \cdot \frac{M a_{1}}{M a} \sqrt{\frac{(\kappa-1) \cdot M a_{1}^{2}+2}{(\kappa-1) \cdot M a^{2}+2}}
$$

From the water steam table it is then possible to determine the speed of sound, velocity, dynamic viscosity, density, Reynolds number, friction coefficient and also the required flow rate:

$$
\dot{m}=S \cdot w \cdot \rho
$$

\subsection{Comparison of individual types of flow}

There are several approaches to calculation of steam blows, each having its pros and cons. In Table 1 comparison is provided of individual approaches to the flow rate calculation using various criteria.

Table 1. Comparison of flow rate calculation possibilities for steam blowing.

\begin{tabular}{|c|c|c|c|c|}
\hline Type of flow & $\begin{array}{c}\text { Bernoulli } \\
\text { flow }\end{array}$ & $\begin{array}{c}\text { Isothermal } \\
\text { flow }\end{array}$ & $\begin{array}{c}\text { Drum } \\
\text { balancing }\end{array}$ & Fanno flow \\
\hline $\begin{array}{c}\text { Are friction losses } \\
\text { considered? }\end{array}$ & yes & yes & yes & yes \\
\hline Are local losses considered? & yes & no & yes & no \\
\hline $\begin{array}{c}\text { Is compressible flow } \\
\text { considered? }\end{array}$ & no & no & yes & yes \\
\hline $\begin{array}{c}\text { Is the model suitable to high } \\
\text { velocity flow? }\end{array}$ & no & no & yes & yes \\
\hline $\begin{array}{c}\text { Is it possible to consider } \\
\text { various pipe diameters? }\end{array}$ & yes & no & yes & no \\
\hline $\begin{array}{c}\text { Can the calculation be done } \\
\text { without pressure } \\
\text { measurement on the tablet? }\end{array}$ & no & no & yes & yes \\
\hline $\begin{array}{c}\text { Can the model be used } \\
\text { without additional } \\
\text { measurement on the drum? }\end{array}$ & yes & yes & no & yes \\
\hline
\end{tabular}

From Table 1 it is evident that the most suitable method is the so-called drum balancing method, which, on the other hand, requires more exact and calibrated measurement than that on the steam drum. This measurement is also financially demanding. Thus the most suitable method appears to be the Fanno flow method analysed below.

\section{CFD flow calculation}

For testing the properties of flows modelled using Fanno flow two variants of $5000 \mathrm{~mm}$ pipelines were modelled in the commercial SW Ansys CFX 17.0. In the first case the pipe was of a constant diameter $D=200 \mathrm{~mm}$ and in the second case the pipe diameter was increased to $D=280 \mathrm{~mm}$ over the final length of $1000 \mathrm{~mm}$. The increased diameter at the end of the pipeline is modelled because it is often used in practice to allegedly slow down the flow before entering the free space. 
Behind the pipeline outlet, free space was modelled in the shape of a block $2500 \mathrm{~mm}$ long with base dimensions $2000 \times 2000 \mathrm{~mm}$. Both solved variants are shown in Fig. 1 1. Boundary conditions are set using pressure inlet $\left(p=10\right.$ bar and $\left.\mathrm{t}=250{ }^{\circ} \mathrm{C}\right)$ and pressure outlet $(p=$ $1 \mathrm{bar}$ ), which is in agreement with commonly achieved conditions in the steam drum and at the pipeline outlet. The flow can be considered compressible, viscous, using SST k- $\omega$ turbulence model of second order accuracy. As a working medium overheated stem was set. The computational mesh was fully structured and contained about 9 million hexahedral cells.
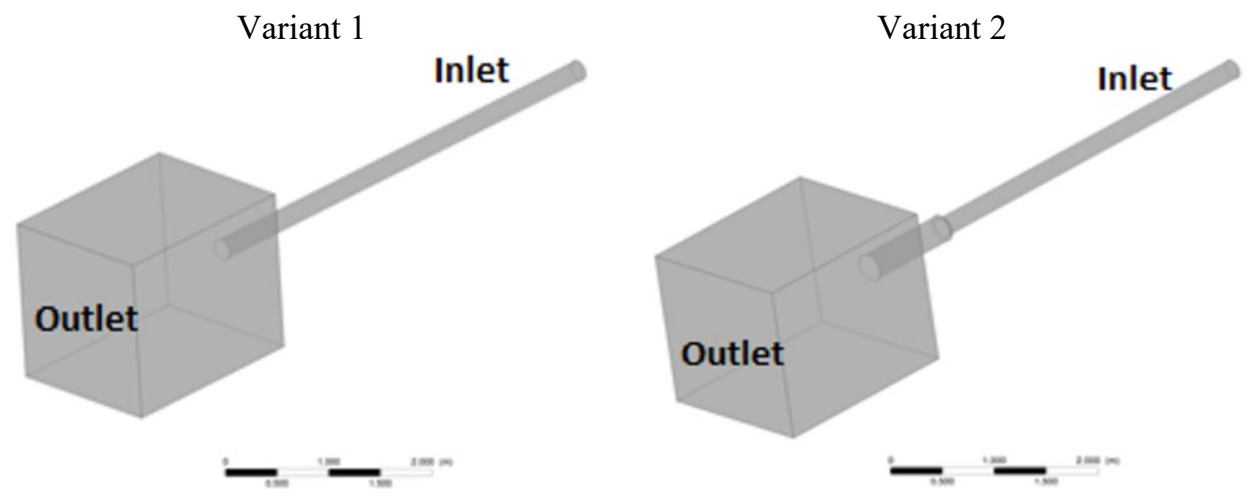

Fig. 1. Two geometrical alternatives of calculated geometry.

\section{Results}

The main results include determination of flow field in the blowing pipeline and comparison of flow parameters distribution along the pipeline determined using CFD calculation and the analytical Fanno flow model. In Figure 2 Mach number distribution is shown for both variants. It is evident that sound flow occurs at the pipeline outlet into the free space and goes on in the free space behind the outlet in the system of oblique sound waves. Even for rather insignificant changes in the pipe diameter at the end of the pipeline the sound flow moves to end section with a larger diameter. Then the flow is already supersonic. The increasing pipe diameter thus does not take the required flow slowing effect, but the pipe end section can be used for silencer installation.

\section{Variant 1}

Mach Number

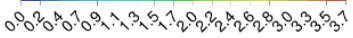
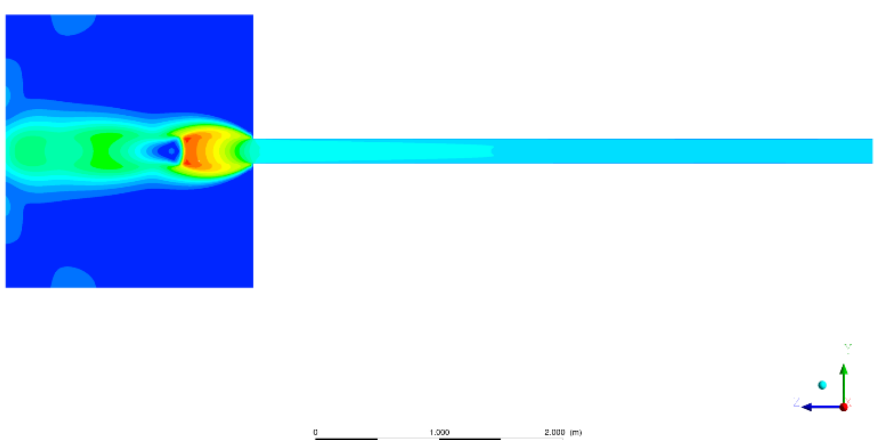


\section{Variant 2}

Mach Number

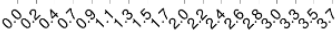

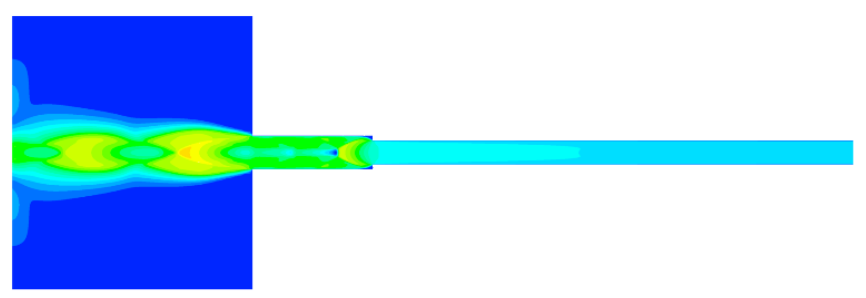

Fig. 2. Mach number flow field.

In Figure 3 distribution of density flow field along the pipeline is shown. For Variant 1 the density along the pipeline decreases steadily and at the pipeline outlet its value is comparable to that of the ambient air. For Variant 2 a significant drop in density occurs at the increased pipe diameter, the supersonic area follows and then flowing into the free space.

Variant 1

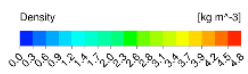

Variant 2

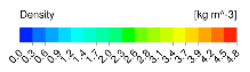

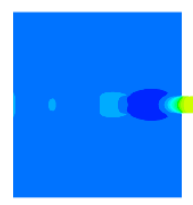

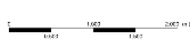

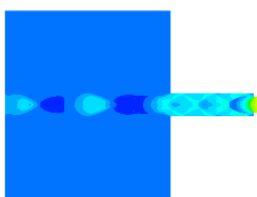

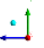

Fig. 3. Density flow field.

In Table 2 the comparison of flows is presented defined using the Fanno flow model and CFD (mass weighted average). The differences between the flow rates are not large; however, the theoretical flow model indicates a higher value of flow rate that is at the increased diameter pipeline significantly closer to the value defined using CFD calculation. The flow rate is thus influenced by the length of the pipeline with constant diameter. With the increasing pipeline length a more significant growth of boundary layers occurs, see Figure 3, the effective cross-section decreases and energetic losses increase.

Table 2. Comparison of flow rates.

\begin{tabular}{|c|c|c|}
\hline & Variant 1 & Variant 2 \\
\hline Fanno $[\mathrm{kg} / \mathrm{s}]$ & 55.9 & 55.9 \\
\hline CFD [kg/s] & 53.9 & 55.5 \\
\hline Relative difference [\%] & 96.4 & 99.3 \\
\hline
\end{tabular}


In the following graphs, courses will be compared of important quantities depending on the pipe length for both variants determined in the case of CFD as mass weighted average.

As the first one in Figure 4 the course is given of static pressure. There are deviations between Fanno flow and Variant 1 that increase along the pipeline. Significant deviation from the model flow has Variant 2, where the effect of expansion which occurred at the distance of $4500 \mathrm{~mm}$ from the inlet is already apparent in the length of $4000 \mathrm{~mm}$. In the final $1000 \mathrm{~mm}$ there is a significant pressure drop almost to the outlet pressure level; in the section of increased diameter of Variant 2 pressure changes no longer occur.

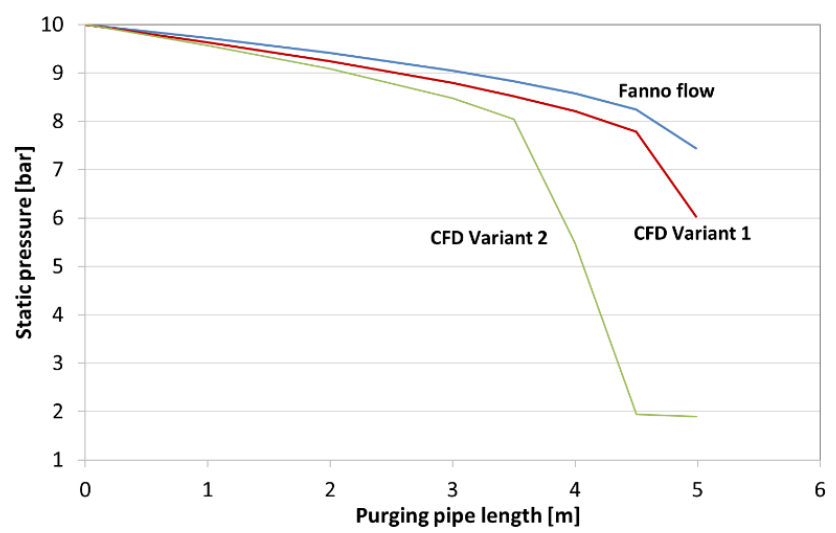

Fig. 4. Courses of static pressure along the pipeline.

The course of Mach number is shown in Figure 5. Again there is a good agreement between Fanno flow and Variant 1 that even further increases in the flow direction. Mach number for Variant 2 is beginning to be affected by diameter increase somewhere between 3500 and $4000 \mathrm{~mm}$ from the inlet. At the distance of $4500 \mathrm{~mm}$ the flow is highly supersonic and enters the free space at a high speed. As a result the blows cause significant noise load on the surroundings.

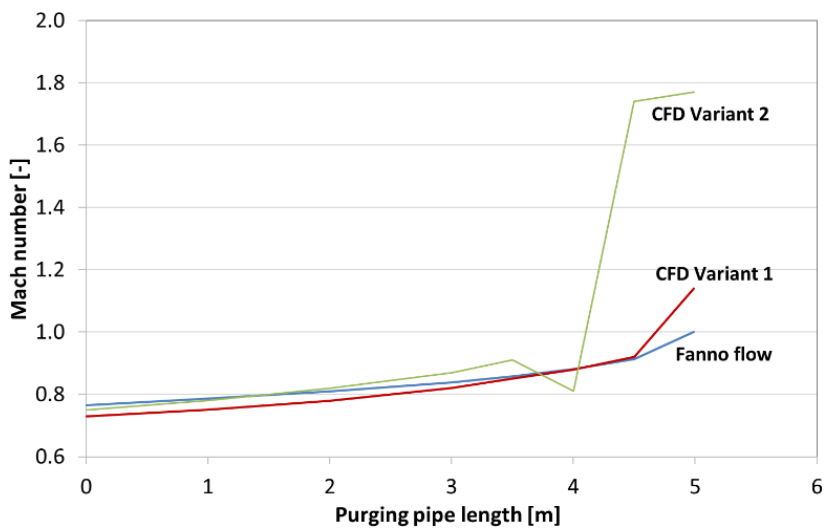

Fig. 5. Mach number courses along the pipeline.

The last graph in Figure 6 shows the courses of static temperatures along the pipeline. Both variants calculated using CFD indicate faster steam cooling than that predicted by Fanno flow. In all cases adiabatic walls are considered. The temperature drop is related to the static pressure, it is the effect of expansion in the narrowing channel. This narrowing is caused by reduction of effective flow cross-section along the pipeline. However, Fanno flow cannot simulate this phenomenon. In this respect the analytical model is rather inaccurate and 
this inaccuracy increases with increasing length of the pipeline. In practice, however, there are many elbows, valves and cross sectional changes that interfere with boundary layers and suppress this phenomenon. On the other hand Fanno flow cannot consider these local losses.

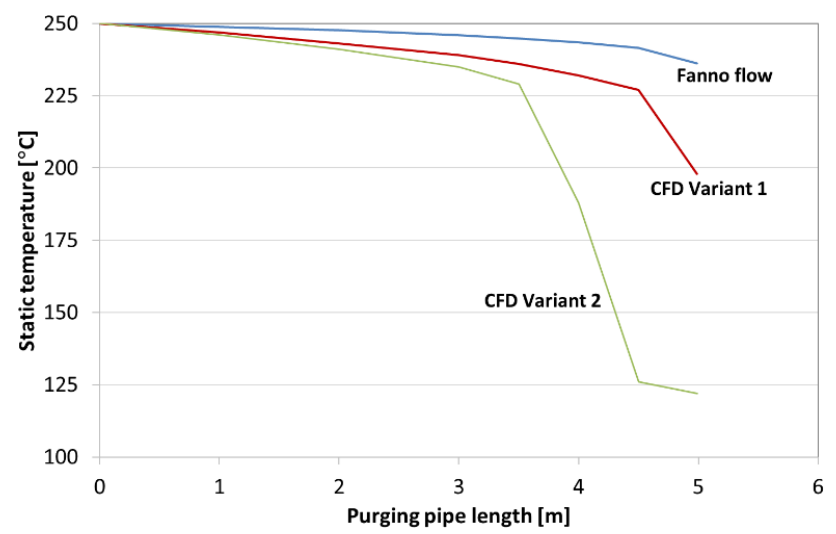

Fig. 6. Temperature courses along the pipeline.

\section{Results}

In the submitted work an overview is presented of analytical methods used for calculation of flow mass during the steam blow. The so-called Fanno flow method was chosen as the most suitable one for comparison with CFD calculations. CFD calculations are carried out for pipes of constant cross-section and for pipes with outlet diameter increase. For pipes with $S=$ const. the sonic throat is located at the pipe outlet to the atmosphere. If the cross section extends the sonic throat moves to the end section and the flow is influenced to the depth of maximum $500 \mathrm{~mm}$; the flow behind the extension is highly supersonic.

The Fanno flow model appears to be promising for use in quick flow rate calculations, but with increasing straight pipe length its accuracy decreases due to the formation of boundary layers and the reduction of effective cross-section flow. Boundary layers are in fact disrupted by many inner struts and local resistances. In this case Fanno flow will provide good results for defining the value of Cleaning Force Ratio without having to install any additional measurements and with preserving a low price of the temporary blowing.

\section{References}

1. Jarvis, Julie \& Babel, Paul \& Vieira, Allen. (2004). Advances in Power Plant Steam Blow Cleaning Analyses. 10.1115/GT2004-53161

2. Jarvis, Julie \& Vieiria, Allen \& Babel, Paul \& Kochis, Paul. (2008). Eliminating Steam Blow Piping Overcleaning During Power Plant Startup. 10.1115/POWER2008-60129

3. Brenner, Matthew J., Babel, Paul J., Jarvis, Julie M., Vieira, Allen T., and Singh, Jyoti. "Steam Blowing Techniques for Large Solar Thermal Power Plants." Proceedings of the ASME 2014 Pressure Vessels and Piping Conference. Volume 3: Design and Analysis. Anaheim, California, USA. July 20-24, 2014. V003T03A038. ASME. https://doi.org/10.1115/PVP2014-29065Brenner, Matthew J., Babel, Paul J., Jarvis, Julie M., Vieira, Allen T., and Singh, Jyoti. "Steam Blowing Techniques for Large Solar Thermal Power Plants." Proceedings of the ASME 2014 Pressure Vessels and Piping Conference. Volume 3: Design and Analysis. Anaheim, California, USA. July 20-24, 2014. V003T03A038. ASME. https://doi.org/10.1115/PVP2014-29065 